\title{
Correction to: Surgical treatment of traumatic distal anterior cerebral artery aneurysm: a report of nine cases from a single centre
}

\author{
Yue He $^{1} \cdot$ Ling Wang $^{2} \cdot$ Yibo Ou ${ }^{1} \cdot$ Heping Wang ${ }^{1} \cdot$ Sheng Wang $^{1} \cdot$ Po Zhang $^{1} \cdot$ Xiaobing He $^{3} \cdot$ Dongsheng Guo $^{1}$
}

Published online: 13 December 2019

(C) Springer-Verlag GmbH Austria, part of Springer Nature 2019

\section{Correction to: Acta Neurochirurgica}

https://doi.org/10.1007/s00701-019-04121-x

The name of Dongsheng Guo was incorrectly captured in the original manuscript.

The correct authorname should be:
$<$ GivenName $>$ Dongsheng $<$ /GivenName $>$ $<$ FamilyName $>$ Guo $<$ /FamilyName $>$ The original article has been corrected.

The online version of the original article can be found at https://doi.org/ 10.1007/s00701-019-04121-x

Dongsheng Guo

Tjguodongsheng@163.com

1 Department of Neurosurgery, Tongji Hospital, Tongji Medical College, Huazhong University of Science and Technology, Wuhan 430030, HuBei Province, People's Republic of China

2 Department of Operating Room, Tongji Hospital, Tongji Medical College, Huazhong University of Science and Technology, Wuhan 430030, HuBei Province, People's Republic of China

3 Department of Neurosurgery, Jianghan Oilfield Central Hospital, Qianjiang 433100, HuBei Province, People's Republic of China 